\title{
ABCB1 C3435T Gene Polymorphism Frequency and Correlation with Clinical Parameters in Multiple Sclerosis
}

\author{
Eda Balkan ${ }^{1 *}$ and Nuray Bilge ${ }^{2}$ \\ ${ }^{1}$ Department of Medical Biology, Ataturk University, Erzurum, Turkey \\ ${ }^{2}$ Department of Neurology, Ataturk University, Erzurum, Turkey
}

\begin{abstract}
Background: People with Multiple Sclerosis (MS) show varying responses to the same drugs, suggesting a genetic factor. In addition, certain $A B C B 1$ gene polymorphisms have been associated with resistance to many drugs. In the era of individualized treatment, identifying possible genetic causes of drug non responsiveness in MS patients may enable the prediction of nonresponse before treatment. Research is needed to determine relationships between $A B C B 1$ polymorphisms and patients' clinical parameters and drug response in MS.
\end{abstract}

Objective: This study investigated the presence of $A B C B 1$ C3435T polymorphism among patients with MS and evaluated possible associations between C3435T variants and disease activity and clinical parameters in MS.

Materials and Methods: The study included 100 patients aged 18 and over who were definitively diagnosed with MS according to the $2010 \mathrm{McDonald}$ diagnostic criteria and were receiving immunomodulatory therapy, as well as a group of 100 healthy individuals. Clinical and demographic characteristics of the MS group were recorded. All study participants underwent $A B C B 1$ C3435T genotyping. A blood sample was collected from each participant and used for DNA isolation and single-nucleotide polymorphism analysis.

Results: There was no statistically significant difference between MS patients and the healthy subjects with regard to $A B C B 1$ C3435T variants. Mean score on the Extended Disability Status Scale was significantly higher in MS patients with the $C T$ variant of the $A B C B 1$ polymorphism compared to those with $C C$ and TT variants, indicating that disability was more severe in MS patients with the CT genotype of the ABCB1 C3435T polymorphism.

Conclusion: Considering the role of P-glycoprotein in drug pharmacokinetics, the results of this study suggest a possible benefit of assessing MS patients for $A B C B 1$ gene polymorphisms. The literature includes very little information on this topic. Although the number of patients in this study was limited, the higher level of disability in MS patients heterozygous for the $A B C B 1$ C $3435 T$ polymorphism is a novel contribution to the literature.

Keywords: $A B C B 1$ gene; C3435T; Disability; Multiple sclerosis; Polymorphism

\section{Statement of Significance}

Multiple Sclerosis (MS) is a debilitating and heterogeneous disease whose management continues to present major challenges. People with MS exhibit a wide range of clinical presentations as well as varying responses to the same drugs, which both indicate that genetic factors are involved in disease course and treatment response. The ability to predict treatment response in MS patients would prevent unnecessary drug usage, limit adverse side effects, and enable earlier direction of patients to effective treatment methods. The $A B C B 1$ gene, which encodes P-glycoprotein 1 , has been associated with resistance to many drugs. While the literature includes studies investigating the role of $A B C B 1$ polymorphisms in other autoimmune diseases, there is little information available regarding its relevance in MS. Therefore, the present study evaluated associations between the ABCB1 C3435T polymorphism and MS patients' clinical parameters such as disease activity and drug changes. The results showed that the heterozygous CT genotype of $A B C B 1$ was associated with significantly higher disability scores, as well as a trend toward higher annual relapse rate and fewer drug changes. This suggests that $A B C B 1$ may have an important role in MS that warrants further research in order to make progress toward individualized therapy for people with MS.

\section{Introduction}

Multiple Sclerosis (MS) is an autoimmune inflammatory demyelinating neurodegenerative disease of Central Nervous System
(CNS) with relapsing-remitting and progressive forms [1]. MS is more common in young adults and women. Age of onset is between 20 and 40 in approximately $65 \%$ of patients. Prevalence studies have shown that the incidence is 2-3 times higher among women than men [2]. The prevalence of MS, which is known to be affected by genetic and environmental factors, varies in different regions. It is considered that this variation is caused by different seasonal characteristics, geographic locations, and ethnic profiles of the population [3]. Although the prevalence and incidence of MS in Turkey have not been definitively determined, it is likely a medium-risk region. A prevalence of 101.4 per 100,000 was reported based on clinical observations [4]. Although its etiology remains unclear, MS is believed to be affected by genetic, environmental, viral, and autoimmune factors [5].

Because inflammation in MS can appear throughout the brain,

*Corresponding author: Dr. Eda Balkan, Department of Medical Biology, Ataturk University, Erzurum, Turkey, Tel: +90-05335402826; Fax: +9004423442301; Email: edadiyarbakir@atauni.edu.tr

Received December 14, 2018; Accepted December 27, 2018; Published December 31, 2018

Citation: Balkan E, Bilge N (2018) ABCB1 C3435T Gene Polymorphism Frequency and Correlation with Clinical Parameters in Multiple Sclerosis. J Neurol Neurophysiol 9: 479. doi:10.4172/2155-9562.1000479

Copyright: ( 2018 Balkan E, et al. This is an open-access article distributed under the terms of the Creative Commons Attribution License, which permits unrestricted use, distribution, and reproduction in any medium, provided the original author and source are credited. 
spinal cord, and optic nerve, it can manifest with any symptom related to the central nervous system. Common signs and symptoms include weakness in the extremities, sensory symptoms, ataxia, bladder problems, fatigue, visual symptoms such as diplopia and blurred vision, dysarthria, and cognitive symptoms such as memory, concentration, and attention disorders [6].

MS is currently diagnosed using the McDonald criteria. These criteria were first introduced in 2001 and revised in 2005, 2010, and 2017. Diagnosis is made based on clinical symptoms and findings. Supportive laboratory findings are obtained through analysis of Magnetic Resonance Imaging (MRI), Cerebrospinal Fluid (CSF), and Visual Evoked Potentials (VEP) [7-9].

MS has several forms which vary in terms of disease course. The most common form encountered in clinical practice is Relapsing Remitting MS (RRMS). Approximately $80 \%-90 \%$ of patients present with RRMS [10]. Primary Progressive MS (PPMS), which accounts for about $10 \%-15 \%$ of cases, is characterized by progressive worsening of symptoms without acute relapses [11]. In Secondary Progressive MS (SPMS), neurodegeneration is more predominant than the inflammatory process. Seventy-five percent of RRMS patients naturally progress to SPMS [12]. An extremely small proportion of patients exhibit a chronically progressive course with acute relapses but no recovery between, a form called Relapsing Progressive MS (RPMS) $[13,14]$.

There is currently no treatment to cure or prevent MS. Treatment options can be grouped into four subheadings: treatment of acute relapses, symptomatic treatment, disease-modifying therapy, and rehabilitation. Acute relapses are treated using corticosteroids and, less frequently, Adrenocorticotrophic Hormone (ACTH). Disease Modifying Therapies (DMTs) including immune-modulatory and immunosuppressant drugs delivered by injection, infusion, and oral route to slow down the progress of MS and to improve patients' quality of life [1-10]. Most of these drugs were approved for the treatment of RRMS. Only mitoxantrone is also approved for the secondary progressive and relapsing progressive forms and ocrelizumab for PPMS.

Patients treated with Interferon-beta (IFN- $\beta$ ) and natalizumab may develop Neutralizing antibodies (NAbs) that can adversely affect therapeutic response. A study revealed that MS patients treated with IFN- $\beta$ who had persistent NAb positivity exhibited more frequent disease activity [15].

Treatment is less effective in NAb-positive patients. Other than this factor, there is no other predictive indicator for DMT response. Identifying the reasons underlying nonresponse to DMTs and predicting which patients will benefit from treatment can both improve medical outcomes and reduce expenses. Genetic variations such as $A B C B 1$ polymorphism may serve as possible predictors of treatment failure [15].

Carrier proteins are very large protein families that transport various drugs, xenobiotics, and endogenous compounds across membranes. They have recently attracted attention because of their role in antineoplastic drug resistance and their effects on drug pharmacokinetics [16].

Numerous drugs are substrates of P-glycoprotein 1 (P-gp). P-gp has a major impact on drug distribution due to its role in the blood-brain barrier and blood-CSF barrier, where it prevents the passage of drugs to the brain and CSF and transports substrates back into the bloodstream [16-18].
Inter-individual variations in drug responses are attributed to polymorphisms or rare phenotypes [19]. The carrier protein that best demonstrates the effect of polymorphisms on pharmacokinetics is P-gp, encoded by the $A B C B 1$ gene. Of the 29 Single-nucleotide Polymorphisms (SNPs) identified for $A B C B 1, \mathrm{C} 3435 \mathrm{~T}$ in exon 26 and G2677T in exon 21 alter the substrate specificity of P-gp to eliminate its carrier protein function. The C3435T and G2677T SNPs may show ethnic variation and imbue resistance to drugs that are substrates of $\mathrm{P}$-gp, thus influencing treatment responses and the prevalence of some diseases [20].

Studies have demonstrated a possible correlation between $A B C B 1$ polymorphism and non-responsiveness to certain drugs. There are also numerous studies regarding $A B C B 1$ polymorphism in relation to autoimmune diseases, immunosuppressant drugs used after transplantation, the determination of psychiatric drug levels, and nonresponsiveness to drugs [16,21-23].

In light of these data, this study was conducted to determine the frequency of the $A B C B 1 \mathrm{C} 3435 \mathrm{~T}$ polymorphism in $100 \mathrm{MS}$ patients being treated with various drugs with different mechanisms of action, and compare them with those of 100 healthy controls. The objective of the study was to identify potential correlations between C3435T variants and disease activity and clinical parameters in MS patients.

\section{Materials and Methods}

This study was jointly planned by the Medical Biology and Neurology Departments of Atatürk University in Erzurum, Turkey. One hundred MS patients aged 18 and over who presented to the MS outpatient clinic of the Erzurum Atatürk University Medical School Neurology Department were included in the study. All patients were diagnosed according to the $2010 \mathrm{McD}$ onald diagnostic criteria and were taking drugs with various mechanisms of action. One hundred healthy, unrelated individuals who had no systemic disease and were not taking any form of medication were included as a control group. Demographic and clinical data pertaining to the patients were noted, including age, gender, and clinical findings such as age at MS onset, disease duration, total number and annual rate of relapses, and Extended Disability Status Scale (EDSS). The patients' current treatment regimens were not modified for the study.

The study protocol was carried out in accordance with the 1989 revised Declaration of Helsinki and was approved by the Ethics Committee of the Erzurum Regional Training and Research Hospital.

\section{Genomic DNA extraction}

Genomic DNA isolation was done using whole blood samples (2cc) collected from each patient into EDTA tubes) using a commercial kit (EZ1 DNA Blood Kit; Qiagen, Germany). DNA was extracted from 200 $\mu \mathrm{L}$ aliquots of whole blood according to the manufacturer's protocol. DNA concentration was diluted to $10 \mathrm{ng} / \mu \mathrm{L}$ for working solutions and the isolated DNA was stored at $-20^{\circ} \mathrm{C}$.

\section{ABCB1 polymorphism analysis}

The present study focused on one Single-nucleotide Polymorphism (SNP) of the ABCB1 gene: C3435T (rs1045642). Polymerase Chain Reaction (PCR) and melting curve analyses were performed under the same conditions in a 96-well plate using a Light Cycler 480 (Roche Diagnostics, Penzberg, Germany). Genotyping was done with Light SNiP typing assay (TIB-MolBiol, Berlin, Germany) by analyzing melting curves with the LightCycler 480 II system (Roche Applied Science, Mannheim, Germany). Samples with a final volume of $20 \mu \mathrm{L}$ were 
prepared by combining $2 \mu \mathrm{L}$ of purified genomic DNA ( $\sim 50 \mathrm{ng}), 2 \mu \mathrm{L}$ of Fast Start DNA Master HybProbe (Roche Diagnostics, Mannheim, Germany), $1 \mu \mathrm{L}$ of Light SNiP Reagent Mix (TIB-MolBiol, Berlin, Germany), $1.6 \mu \mathrm{L}$ of $25 \mathrm{mM} \mathrm{MgCl}_{2}$, and $13.4 \mu \mathrm{L}$ of distilled $\mathrm{H}_{2} \mathrm{O}$. Realtime PCR was performed as follows: denaturation at $95^{\circ} \mathrm{C}$ for $10 \mathrm{~min}$, followed by 45 cycles of $95^{\circ} \mathrm{C}$ for $10 \mathrm{~s}, 60^{\circ} \mathrm{C}$ for $10 \mathrm{~s}$, and $72^{\circ} \mathrm{C}$ for $15 \mathrm{~s}$. After the amplification phase, a melting curve analysis was performed at $95^{\circ} \mathrm{C}$ for $30 \mathrm{~s}, 40^{\circ} \mathrm{C}$ for $2 \mathrm{~min}, 75^{\circ} \mathrm{C}$ for $0 \mathrm{~s}$, followed by cooling phase at $40^{\circ} \mathrm{C}$ for $30 \mathrm{~s}$. Collected data were analysed using LightCycler 480 Gene Scanning software version 1.2 (Roche Diagnostics).

\section{Statistical Analysis}

The study data were analysed using SPSS ${ }^{\bullet}$ version 23.0 (IBM Corp., Armonk, NY, USA) statistical software package. Frequency distribution, mean, and standard deviation values were used for comparisons between the groups. Kolmogorov-Smirnov test was used to assess normality of data distributions. Kruskal-Wallis variance of analysis was used to compare number of drug changes and annual relapse rate between polymorphism groups due to the non-normal distribution of the data. ANOVA was used for comparison of Extended Disability Status Scale (EDSS) scores because the data were normally distributed.

\section{Results}

The study included 100 MS patients and 100 healthy individuals

\begin{tabular}{|l|c|c|}
\hline ABCB1 3435C>T Haplotype & MS $^{*}(\mathbf{n})$ & Control $(\mathbf{n})$ \\
\hline CC & 23 & 21 \\
\hline CT & 46 & 40 \\
\hline TT & 31 & 34 \\
\hline Total & 100 & 100 \\
\hline
\end{tabular}

Table 1: Distribution of C3435T genotypes and allele frequencies in MS patients and controls ${ }^{*} \mathrm{MS}$ : Multiple sclerosis.

in the control group, and investigated one SNP in a single gene. The mean age of the patients was $36.41 \pm 9.53$ years and the male to female ratio was 1:2.6. Frequencies of $A B C B 1$ rs: 1045642 (C3435T) variants in the patient and control groups are summarized in Table 1. In the RRMS group, the heterozygous CT genotype was the most common, present in 38 patients, followed by the ho Of the MS patients who were included in the study group, 63 used DMT drugs (Interferon betala, Interferon beta 1b, Glatiramer Acetate), 30 oral (Teriflunamide, Dimethyl Fumarate, Fingolimod) treatment and 7 patients were using Natalizumab (300 $\mathrm{mg}$, iv). When the relationship between drug groups and $A B C B 1$ polymorphism $3435 \mathrm{C}>\mathrm{T}$ was examined, all of the treatment groups had more CT variants. Mozygous TT genotype in 28 patients and homozygous CC genotype in 21 patients. The patients' demographic and clinical characteristics and drugs used are presented in Table 2.

The MS patients were comparedby $A B C B 1$ C3435T genotype in terms of EDSS score, age at disease onset, duration of disease, annual relapse rate, and number of drug changes (Table 3). Annual relapse rate was highest among the MS patients with the CT genotype (0.97 \pm 0.01 ), but the difference between the groups was not statistically significant $(\mathrm{p}<0.05)$ (Figure 1) (Table 4$)$. Number of drug changes was lowest in patients with the CT genotype and highest in those with the CC genotype, but there was no statistically significant difference between the groups $(\mathrm{p}<0.05)$ (Figure 1). Mean EDSS scores by polymorphism group were 2.48 in CT, 1.89 in TT, and 1.80 in CC. EDSS was significantly correlated with polymorphism $(\mathrm{p}<0.05)$. Paired comparisons between groups showed statistically significant differences in EDSS between the CT and CC groups and between the $\mathrm{CT}$ and TT groups $(\mathrm{p}<0.05)$.

\section{Discussion}

Although its etiology of MS is not well understood, it is considered

\begin{tabular}{|c|c|c|c|c|}
\hline & $\begin{array}{c}\text { TT } \\
(n=31)\end{array}$ & $\begin{array}{c}C C \\
(n=23)\end{array}$ & $\begin{array}{c}C T \\
(n=46)\end{array}$ & Total $(n=100)$ \\
\hline Male/Female & $1: 4.43$ & $1: 2.55$ & $1: 3.83$ & $1: 2.6$ \\
\hline Age, years (mean $\pm S D$ ) & $35.13 \pm 8.10$ & $38.22 \pm 11.69$ & $36.37 \pm 9.30$ & $36.41 \pm 9.53$ \\
\hline Age at onset, years (mean $\pm S D$ ) & $30.95 \pm 8.20$ & $31.96 \pm 9.17$ & $28.78 \pm 9.00$ & $30.19 \pm 8.81$ \\
\hline Duration of disease, years (mean $\pm \mathrm{SD}$ ) & $4.18 \pm 2.18$ & $6.26 \pm 5.79$ & $7.59 \pm 6.54$ & $6.23 \pm 5.34$ \\
\hline Annual relapse rate (mean $\pm S D$ ) & $0.86 \pm 0.01$ & $1.02 \pm 0.03$ & $0.97 \pm 0.01$ & $2.85 \pm 0.02$ \\
\hline Number of drug changes (mean $\pm \mathrm{SD}$ ) & $0.46 \pm 0.01$ & $0.49 \pm 0.01$ & $0.42 \pm 0.01$ & $1.38 \pm 0.01$ \\
\hline EDSS (mean \pm SD) & $1.89 \pm 0.01$ & $1.80 \pm 1.31$ & $2.48 \pm 1.32$ & $2.14 \pm 1.26$ \\
\hline \multicolumn{5}{|l|}{ Type of multiple sclerosis } \\
\hline RRMS & 28 & 21 & 38 & $87(87 \%)$ \\
\hline SPMS & 2 & 2 & 8 & $12(12 \%)$ \\
\hline PPMS & 1 & 0 & 0 & $1(1 \%)$ \\
\hline
\end{tabular}

Table 2: Demographic and clinical characteristics of multiple sclerosis patients genotyped for $A B C B 1$.

\begin{tabular}{|c|c|c|c|c|}
\hline Drugs used by multiple sclerosis patients as disease-modifying therapy & Total patient number $(n=100)$ & TT $(n=31)$ & CT $(n=46)$ & $C C(n=23)$ \\
\hline Interferon beta $1 \mathrm{a}(\mathrm{sc}) 22 \mu \mathrm{g}$ or $44 \mu \mathrm{g}$ dose 3 times weekly & 31 & 14 & 12 & 5 \\
\hline Interferon beta $1 \mathrm{a}(\mathrm{im}) 30 \mu \mathrm{g}$ once weekly & 9 & 2 & 4 & 3 \\
\hline Interferon beta $1 \mathrm{~b} 20 \mathrm{mg} /$ day or $40 \mathrm{mg} 3$ times/week & 12 & 2 & 6 & 4 \\
\hline \multicolumn{5}{|l|}{ (sc) $250 \mu g$ every other day } \\
\hline Glatiramer acetate & 11 & 4 & 5 & 2 \\
\hline Dimethyl fumarate oral $240 \mathrm{mg}$ twice daily & 9 & 2 & 4 & 3 \\
\hline Teriflunomide Oral 7-14 mg/day & 7 & 2 & 3 & 2 \\
\hline Fingolimod Oral 0.5 mg/day & 14 & 3 & 9 & 2 \\
\hline Natalizumab (iv) 300 mg once monthly & 7 & 2 & 3 & 2 \\
\hline
\end{tabular}

Table 3: Drugs used by MS patients as disease-modifying therapy. 


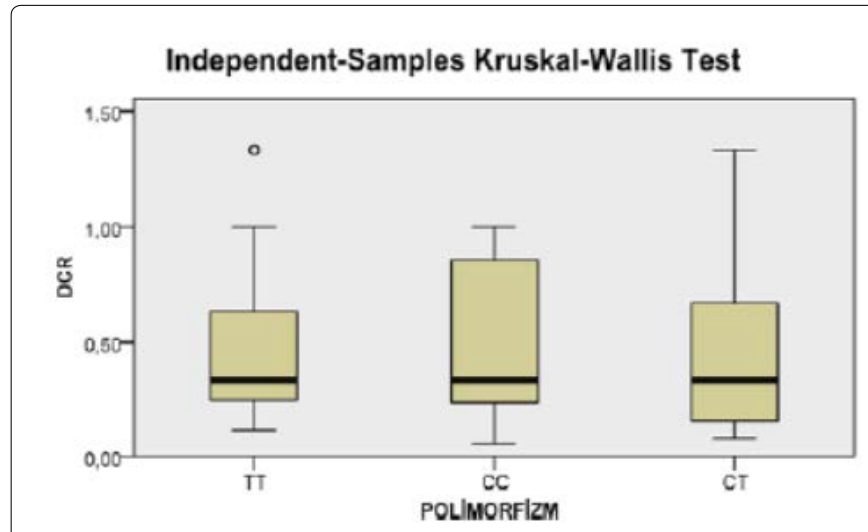

Independent-Samples Kruskal-Wallis Test

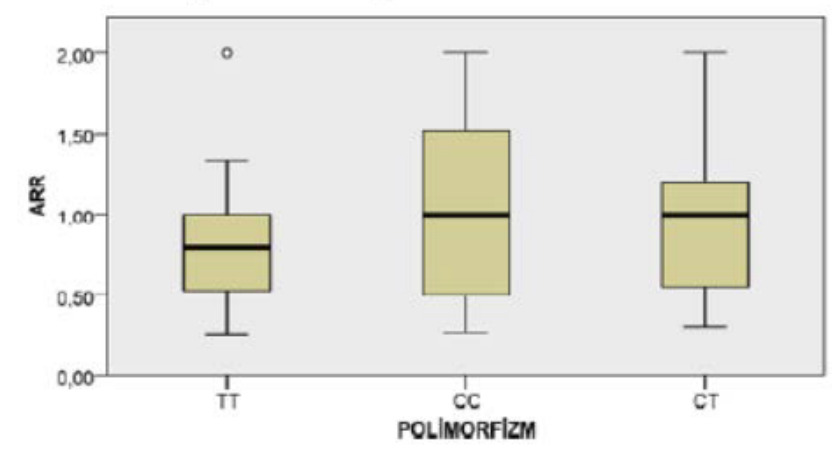

Figure 1: Comparison of number of Drug Changes (DCR) and Annual Relapse Rate (ARR) between $A B C B 1$ polymorphism groups.

\begin{tabular}{|l|c|}
\hline Clinical parameters & $\begin{array}{c}A B C B 1 \text { genotype TT-CT-CC } \\
\text { Polymorphism (Kruskal-Wallis test) }\end{array}$ \\
\hline Annual relapse rate & $p=0.56$ \\
\hline Number of drug changes & $p=0.61$ \\
\hline
\end{tabular}

Table 4: Statistical comparison of annual relapse rate and number of drug changes of multiple sclerosis patients based on $A B C B 1$ polymorphism genotype (Significant at the $p<0.05$ level, highly significant at $p<0.01^{*}$ (Kruskal-Wallis test)).

to be an autoimmune disease related to both genetic and environmental factors [24]. This study presents an analysis of C3435T polymorphic variants of the $A B C B 1$ gene in patients with MS and demonstrates a novel association between $A B C B 1$ genotype and patients' disability scores as measured by the EDSS.

P-gp is an ATP-dependent efflux pump that consists of 280 amino acids and has a molecular weight of $170 \mathrm{kDa}$. It is known that altering P-gp expression and function can change the absorption and tissue distribution its substrate drugs, thereby influencing treatment response [25].

The effect of $A B C B 1$ gene polymorphisms in different diseases is not clear. This is the principal reason for investigating each $A B C B 1$ SNP separately. The literature includes many studies on drug resistance and $A B C B 1$ gene polymorphism. These studies evaluated associations between $A B C B 1$ polymorphisms in various autoimmune diseases including Behçet's disease, systemic lupus erythematous, scleroderma, rheumatoid arthritis, and ankylosing spondylitis. However, few studies have focused on MS [21-23].

The first study demonstrating the relationship between MDR1 $(A B C B 1)$ gene polymorphism and P-gp expression and drug blood levels was found and found that gene polymorphisms affect drug blood levels by determining that drug blood level (digoxin) is higher in patients with TT genotype [26].

Kerb et al. suggested that $A B C B 1$ polymorphism is an important parameter in the development of drug resistance [27]. The C3435T polymorphism is the most common $A B C B 1$ gene polymorphism, resulting from a single base change $(\mathrm{C}-\mathrm{T})$ at position 3435 in exon 26. It does not cause an amino acid change [28].

The effects of $A B C B 1$ SNPs are a newly emerging area of research in MS etiology. No statistical differences have been previously reported between MS patients and controls in terms of $A B C B 12677 \mathrm{G}>\mathrm{T}$ and $3435 \mathrm{C}>\mathrm{T}$. SNPs in ABC transporter genes can function as pharmaco genetic markers associated with clinical response to drug therapy in multiple sclerosis [29].

In the present study, the mean age at MS onset was 19 years, and the patients' mean age was 36.4 years. The male to female ratio was 1:2.6, which is consistent with the literature. Similarly, the most common form of MS in the present study was RRMS, accounting for $87 \%$ of the patients. An additional 12\% of the patients had SPMS and 1\% had PPMS.

\section{Conclusion}

Our study constitutes a small population of MS patients in Turkey. The results of this study show that EDSS values were significantly higher among patients with the CT polymorphism when compared with the other two groups. This suggests that disability is more severe in MS patients having the CT genotype of the $A B C B 1$ polymorphism. Presence of CT polymorphism more extensive and large studies are needed to determine whether MS patients are a predictive parameter for progression. This study may contribute to the literature in that sense. Subsequent studies will investigate larger populations with a broader scope.

\section{Author Contributions}

Study design/planning: Eda Balkan; Data collection/entry: Nuray Bilge; Data analysis/statistics: Nuray Bilge; Data interpretation: Eda Balkan; Manuscrip preparation: Eda Balkan; Literature search/analysis: Eda Balkan; Funds collection: Eda Balkan and Nuray Bilge.

\section{Ethical Standards}

The study was conducted in compliance with international, national, and institutional regulations. The Ataturk University Medical Faculty Ethics Committee approved the study. All persons provided informed consent prior to inclusion in the study.

\section{References}

1. Fazzitol MM, Jordy SS, Tilbery CP (2009) Psychiatric disorders in multiple sclerosis patients. Arc Neuro-Psiquiatr 67: 664-667.

2. Compston A, Coles A (2008) Multiple sclerosis. Lancet 372: 1502-1517.

3. Mirza M (2002) Etiology and epidemiology of multiple sclerosis. Erciyes Medical Journal 24: 40-47.

4. Börü ÜT, Alp R, Sur H (2006) Prevalence of multiple sclerosis door-todoor survey in Maltepe, Istanbul, Turkey. Neuroepidemiology 27: 17-21.

5. Hafler DA, Slavik JM, Anderson DE, O'Connor KC, De Jager P, et al. (2005) Multiple sclerosis. Immunological reviews 204: 208-231.

6. Cavit B (2009) Clinical findings and symptoms in multiple sclerosis. Turkiye Klinikleri Journal of Neurology Special Topics 2: 9-15.

7. McDonald WI, Compston A, Edan G, Goodkin D, Hartung HP, et al. (2001) Recommended diagnostic criteria for multiple sclerosis: Guidelines from the International Panel on the diagnosis of multiple sclerosis. Ann Neurol 50: 121127. 
Citation: Balkan E, Bilge N (2018) ABCB1 C3435T Gene Polymorphism Frequency and Correlation with Clinical Parameters in Multiple Sclerosis. J Neurol Neurophysiol 9: 479. doi:10.4172/2155-9562.1000479

8. Polman $\mathrm{CH}$, Reingold SC, Edan G, Filippi M, Hartung HP, et al. (2005) Diagnostic criteria for multiple sclerosis: Revisions to the McDonald Criteria. Ann Neurol 58: 840-846.

9. Polman $\mathrm{CH}$, Reingold SC, Banwell B, Clanet M, Cohen JA, et al. (2011) Diagnostic criteria for multiple sclerosis: Revisions to the McDonald criteria. Ann Neurol 69: 292-302.

10. Kantarci OH, Weinshenker BG (2005) Natural history of multiple sclerosis. Neurol Clin 23: 17-38.

11. Koch M, Kingwell E, Rieckmann P, Tremlett $H$ (2009) The natural history of primary progressive multiple sclerosis. Neurology 73: 1996-2002.

12. Runmarker B, Andersen $O$ (1993) Prognostic factors in a multiple sclerosis incidence cohort with twenty-five years of follow-up. Brain 116: 117-134.

13. Vukusic S, Confavreux C (2010) Natural history of multiple sclerosis. Presse Médicale 39: 359-362.

14. Lublin FD, Reingold SC (1996) Defining the clinical course of multiple sclerosis: Results of an international survey. Neurology 46: 907-911.

15. Allan CB (2018) Multıple sclerosis mechanism and immunotherapy. Neuron 97 742-768.

16. Gül IG, Eryılmaz G, Karamustafalıoglu OK (2016) P-glycoprotein and its role in treatment resistance. Current Approaches in Psychiatry 8: 19-31.

17. Aszalos A (2007) Drug-drug interactions affected by the transporter protein, P-glycoprotein (ABCB1, MDR1) II. Clinical aspects. Drug Discov Today 12: 838-843

18. Balayssac D, Authier N, Cayre A, Coudore F (2005) Does inhibition of P-glycoprotein lead to drug-drug interactions? Toxicol Lett 156: 319-329.

19. Kayaalp SO (2000) Medical pharmacology for rational treatment all 1 and 2 skin. Tıbbi Farmakoloji 9: 96-107.
20. Fromm MF (2002) Genetically determined differences in P- glycoprotein function: Implications for disease risk. Toxicology 5: 181-182.

21. Lee YH, Bae SC, Song GG (2016) Association of the ABCB1 C3435T polymorphism with responsiveness to and toxicity of DMARDs in rheumatoid arthritis: A meta-analysis. Z Rheumatol 75: 707-715.

22. Paul TM, Vikramraj KA, Christina J, Mariaselvam M, Negi S (2015) Multidrug resistance 1 (MDR1) 3435C>T gene polymorphism iinfluences the clinical phenotype and methotrexate-inducedadverse. Eur J Clin Pharmacol 71: 959-996.

23. Capron A, Mourad M, de Meyer M, De Pauw L, Eddour DC, et al. (2010) CYP3A5 and $A B C B 1$ polymorphisms influence tacrolimus concentrations in peripheral blood mononuclear cells after renal transplantation. Pharmacogenomics 11: 703-714.

24. Mirza M (2002) The etiology and the epistemology of the multiple sclerosis Erciyes Med J 24: 40-47.

25. Fojo A, Lebo R, Shimizu N, Chin JE, Roninson IB, et al. (1986) Localization of multidrug resistance-associated DNA sequences to human chromosome. Somat Cell Mol Genet 12: 415-20.

26. Hoffmeyer S (2000) Functinal polymorphisms of the human multidrugresistance gene: Multiple sequence variations and correlation of one allele with pglycoprotein expression and activity in vivo. Proc Natl Acad Sci 97: 3473-3478.

27. Kebr R (2001) ABC drug transporters: Hereditary polymorphisms and pharmacological impact in MDR 1, MRP 1 and MRP 2. Pharmacogenomics 2: $51-64$.

28. Mın DI, Ellıngrod VL (2002) C3438T mutation in exon 26 of the human MDR 1 geneand cyclosporine pharmacokinetics in healthy subjects. Ther Drug Monit 24: 400-404

29. Cotte S, von Ahsen N, Kruse N (2009) ABC-transporter gene-polymorphisms are potential pharmacogenetic markers for mitoxantrone response in multiple sclerosis. Brain 132: 2517-2530. 